\title{
Distressed Communities Index in Patients Undergoing Transcatheter Aortic Valve Implantation in an Affluent County in New York
}

\author{
Thomas Bilfinger $\mathbb{D}^{1},{ }^{1}$ Allison Nemesure, ${ }^{1}$ Robert Pyo, ${ }^{2}$ Jonathan Weinstein, ${ }^{2}$ \\ Giridhar Korlipara, ${ }^{2}$ Daniel Montellese, ${ }^{2}$ Shamim Khan, ${ }^{2}$ Neal Patel, ${ }^{2}$ Henry Tannous, \\ Ting-Yu Wang, ${ }^{2}$ Ely Gracia, ${ }^{2}$ Susan Callahan, ${ }^{2}$ and Puja B. Parikh ${ }^{2}$ \\ ${ }^{1}$ Division of Cardiothoracic Surgery, Department of Medicine, State University of New York at Stony Brook, Stony Brook, \\ NY, USA \\ ${ }^{2}$ Division of Cardiovascular Medicine, Department of Medicine, State University of New York at Stony Brook, Stony Brook, \\ NY, USA
}

Correspondence should be addressed to Thomas Bilfinger; thomas.bilfinger@stonybrookmedicine.edu

Received 13 August 2020; Revised 7 July 2021; Accepted 13 August 2021; Published 24 August 2021

Academic Editor: Thach N. Nguyen

Copyright (c) 2021 Thomas Bilfinger et al. This is an open access article distributed under the Creative Commons Attribution License, which permits unrestricted use, distribution, and reproduction in any medium, provided the original work is properly cited.

\begin{abstract}
Background. The clinical impact of the distressed communities index (DCI), a composite measure of economic well-being based on the U.S. zip code, is becoming increasingly recognized. Ranging from 0 (prosperous) to 100 (distressed), DCI's association with cardiovascular outcomes remains unknown. We aimed to study the association of the DCI with presentation and outcomes in adults with severe symptomatic aortic stenosis (AS) undergoing transcatheter aortic valve intervention (TAVR) in an affluent county in New York. Methods. The study population included 286 patients with severe symptomatic AS or degeneration of a bioprosthetic valve who underwent TAVR with a newer generation transcatheter heart valve (THV) from December 2015 to June 2018 at an academic tertiary medical center. DCI for each patient was derived from their primary residence zip code. Patients were classified into DCI deciles and then categorized into 4 groups. The primary and secondary outcomes of interest were 30 -day, 1year, and 3-year mortality, respectively. Results. Among 286 patients studied, 26\%, 28\%, 28\%, and 18\% were categorized into DCI groups 1-4, respectively (DCI <10: $n=73$; DCI 10-20: $n=81$; DCI 20-30: $n=80$; DCI >30: $n=52$ ). Patients in group 4 were younger with worse kidney function compared to patients in groups 1 and 2 . They also had smaller aortic annuli and were more likely to receive a smaller THV. No significant difference in hospital length of stay or distribution of in-hospital, 30-day, 1-year, and 3-year mortality was demonstrated. Conclusions. While the DCI was associated with differences in the clinical and anatomic profile, it was not associated with differences in clinical outcomes in this prospective observational study of adults undergoing TAVR suggesting that access to care is the likely discriminator.
\end{abstract}

\section{Introduction}

The success of new technology can be measured by the time it takes to penetrate a given market. With high research and development costs, adaptation of new technology is typically occurring along socioeconomic gradients [1]. The clinical impact of the distressed communities index (DCI), a composite measure of economic well-being based on the
U.S. zip code, is becoming increasingly recognized. Ranging from 0 (prosperous) to 100 (distressed), DCI's association with cardiovascular outcomes remains unknown [2]. Transcatheter aortic valve intervention (TAVR), which was introduced over a decade ago, has radically changed how we treat severe symptomatic aortic stenosis (AS) and bioprosthetic aortic valve degeneration [3]. While data are emerging that TAVR programs are concentrated in 
wealthier areas, raising questions about access, no data exist on the association between the DCI and outcomes following TAVR. Accordingly, we aimed to study the association of the DCI with presentation, management, and outcomes in adults with severe symptomatic AS or bioprosthetic valve degeneration undergoing TAVR at an academic tertiary care center in an affluent county in New York.

\section{Methods}

We prospectively included all patients undergoing TAVR at Stony Brook University Hospital, an academic tertiary medical center, in our institutional registry. Adults (age $>18$ years) with severe symptomatic AS and/or failure of a bioprosthetic valve and undergoing TAVR from December 2015 to June 2018 were included in this study.

Suffolk County is the easternmost county on Long Island, New York. It comprises 107 zip codes, each of which comprises at least 500 inhabitants [4]. The DCI is available for all zip codes with more than 500 residents, which captures $99 \%$ of the U.S. population. It is a composite score based on 7 metrics: no high-school degree, housing vacancy rate, adults not working, poverty rate, median income ratio, change in employment, and change in business establishments [2]. The 7 evenly weighted variables are used to calculate a zip code rank compared with its geographic peers and then normalized to obtain a raw distress score that ranges from 0 (no distress) to 100 (severe distress). The 7 socioeconomic status (SES) indicators were obtained from 5-year estimates from the 2014 American Community Survey and the Census Bureau County and ZIP Code Business Pattern. The Economic Innovation Group provides a heat map of DCI scores across the U.S. The number of valves estimated in patients with home zip codes in Suffolk was obtained from Medicare claims data for all 107 Suffolk County zip codes.

Patients were classified into deciles of the DCI based on the zip code of their primary address. For analysis, we grouped DCI values $>30$ together as there were few patients in each decile above 30. Demographic and medical history extracted included age, sex, body mass index (BMI), prior coronary artery bypass graft surgery (CABG), prior aortic valve replacement (AVR), prior balloon aortic valvuloplasty $(\mathrm{BAV})$, prior mitral valve surgery, prior myocardial infarction (MI), atrial fibrillation (AF), prior pacemaker/defibrillator, prior stroke/transient ischemic attack, chronic obstructive pulmonary disease (COPD), obstructive sleep apnea, diabetes mellitus, carotid disease, peripheral arterial disease, and serum creatinine $(\mathrm{mg} / \mathrm{dl})$. The Society of Thoracic Surgeons (STS) predicted the risk of mortality was obtained for each patient. Echocardiographic data extracted included aortic valve area (AVA) and index (AVAI) and left ventricular ejection fraction (LVEF). Aortic annular area and perimeter were also obtained from gated computed tomography angiography (CTA). Procedural data (e.g., access, anesthesia, transcatheter valve size and type, predilatation, and postdilatation) and discharge data (e.g., discharge antiplatelets, discharge anticoagulants, discharge location, length of stay (LOS), and in-hospital and 30-day outcomes (e.g., all-cause mortality, disabling stroke, new pacemaker, and hospital readmission), as well as 1- and 3year all-cause mortality, were also collected. This study was approved by our Institutional Review Board. A waiver of consent to use data prospectively was obtained for all patients.

Continuous variables were presented as mean$s \pm$ standard deviation (SD) and compared using one-way ANOVA. Categorical variables were presented as percentages and compared with the chi-squared test. Histogram of the DCI was performed for the Suffolk County population, our overall study population, and for patients requiring pacemaker, hospital readmission, and/or discharge to a skilled nursing facility. SPSS version 23.0 (SPSS Inc., Chicago, IL) was used for data analysis, and a two-tailed $P$ value of 0.05 was regarded as statistically significant.

\section{Results}

The distribution of DCI scores on Long Island, New York, is skewed towards a low DCI score (Figure 1). Our study population included 286 consecutive patients who underwent TAVR from December 2015 to June 2018 at a single academic tertiary care institution. Among the 286 patients studied, $26 \%, 28 \%, 28 \%$, and $18 \%$ were categorized into DCI groups 1-4, respectively (DCI $<10: n=73$; DCI 10-20: $n=81$; DCI 20-30: $n=80$; DCI >30: $n=52$ ). Patients in group 4 had increased serum creatinine compared to patients in groups 1 and 2 (Table 1). They also had smaller aortic annuli and were more likely to receive a smaller THV (Tables 2 and 3). No difference in age and STS predicted risk of mortality was noted across the 4 groups. With respect to outcomes, no significant difference in hospital length of stay or rate of in-hospital and 30-day mortality, stroke, new pacemaker, and readmission was detected (Table 4). The distribution of patients requiring a skilled nursing facility, new pacemaker, or 30-day readmission followed the DCI histogram of that of the overall TAVR population implanted (Figures 2(a)-2(d)). One-year all-cause mortality rates were also similar among the groups (Figure 3), and at 3 years, $76 /$ $286(26.6 \%)$ had died.

\section{Discussion}

In this contemporary prospective study of adults undergoing TAVR at an academic medical center in an affluent county in New York, several findings are noteworthy. First, patients with worse DCI were younger, had worse kidney function, and were more likely to receive a smaller THV. Second, DCI was not associated with early in-hospital or 30-day outcomes. Finally, 1- and 3-year mortality was similar across the DCI groups. To our knowledge, this is the first study to assess the association between the DCI and outcomes in patients undergoing TAVR, during a time period where TAVR surpassed SAVR in New York state (Table 5). A likely explanation for the absence of postprocedural differences is the intense competition of regional TAVR programs which all perform in or near the top on the composite metric for benchmarking recently published [5]. 


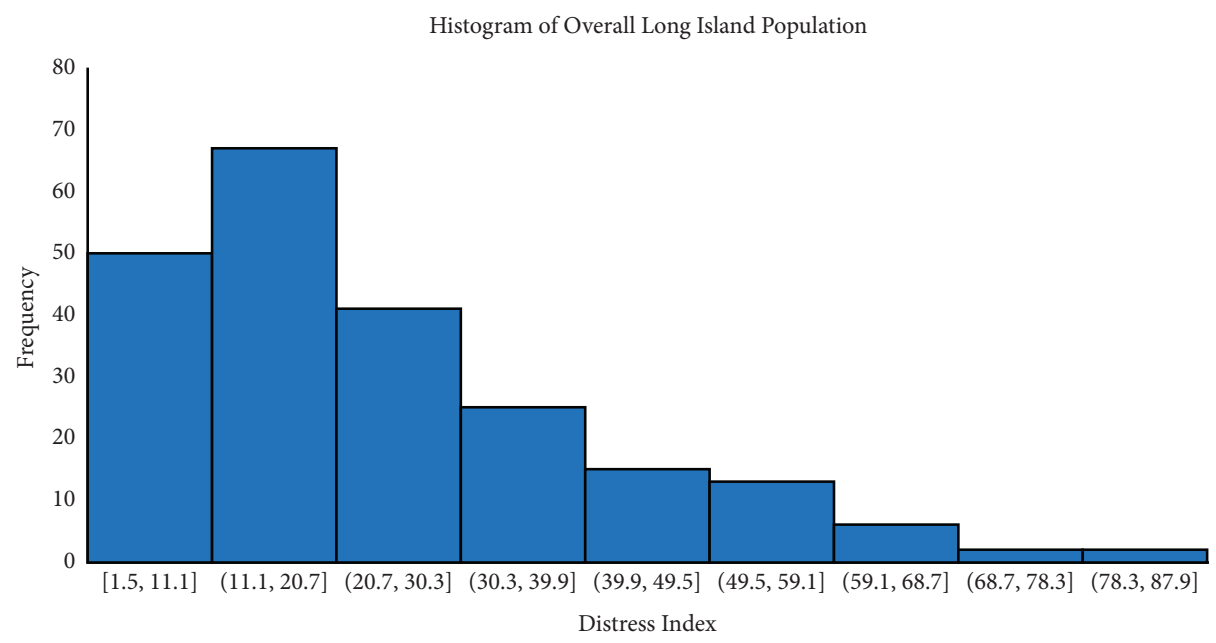

FIgURE 1: Histogram of the distressed communities index across Long Island, New York.

TABLE 1: Baseline medical history.

\begin{tabular}{lcccc}
\hline & $\begin{array}{c}\text { Distress index }<10 \\
(n=73)\end{array}$ & $\begin{array}{c}\text { Distress index } 10-20 \\
(n=81)\end{array}$ & $\begin{array}{c}\text { Distress index 20-30 } \\
(n=80)\end{array}$ & $\begin{array}{c}\text { Distress index }>30 \\
(n=52)\end{array}$ \\
\hline Age (years) & $80 \pm 9$ & $81 \pm 8$ & $79 \pm 8$ & $77 \pm 10$ \\
Female gender & $36(49.3 \%)$ & $35(43.2 \%)$ & $35(43.8 \%)$ & $32(61.5 \%)$ \\
Weight (kg) & $83 \pm 19$ & $80 \pm 19$ & $82 \pm 21$ & $77 \pm 18$ \\
Height (meters) & $1.67 \pm 0.11$ & $1.68 \pm 0.10$ & $1.66 \pm 0.11$ & $1.63 \pm 0.11$ \\
Body mass index $\left(\mathrm{kg} / \mathrm{m}^{2}\right)$ & $29.5 \pm 5.8$ & $28.0 \pm 6.6$ & $29.3 \pm 6.4$ & $27.6 \pm 8.3$ \\
STS predicted risk of mortality (PROM) & $6.4 \pm 5.7$ & $6.4 \pm 4.5$ & $6.7 \pm 4.2$ & $6.1 \pm 5.4$ \\
Serum creatinine (mg/dl) & $1.1 \pm 0.5$ & $1.3 \pm 1.0$ & $1.2 \pm 0.6$ & $1.6 \pm 1.6$ \\
Prior coronary artery bypass grafting & $18(24.7 \%)$ & $18(22.2 \%)$ & $23(28.7 \%)$ & $4(7.7 \%)$ \\
Prior myocardial infarction & $8(11.0 \%)$ & $23(28.4 \%)$ & $7(8.8 \%)$ & $12(23.1 \%)$ \\
Prior aortic value replacement & $1(1.4 \%)$ & $2(2.5 \%)$ & $1(1.3 \%)$ & $3(5.8 \%)$ \\
Prior balloon aortic valvuloplasty & $1(1.4 \%)$ & $4(5.0 \%)$ & $7(8.8 \%)$ & $3(5.8 \%)$ \\
Prior mitral valve surgery & $3(4.1 \%)$ & $0(0 \%)$ & $13(16.3 \%)$ & $0(0 \%)$ \\
Prior pacemaker/defibrillator & $12(16.4 \%)$ & $7(8.6 \%)$ & $28(35.0 \%)$ & $4(7.7 \%)$ \\
Atrial fibrillator & $33(45.2 \%)$ & $33(40.7 \%)$ & $19(23.8 \%)$ & $12(23.1 \%)$ \\
Chronic obstructive pulmonary disease & $12(16.4 \%)$ & $20(24.7 \%)$ & $8(10.0 \%)$ & $10(19.2 \%)$ \\
Obstructive sleep apnea & $6(8.2 \%)$ & $9(11.1 \%)$ & $12(15.0 \%)$ & $2(3.8 \%)$ \\
Prior stroke/transient ischemic attack & $11(15.1 \%)$ & $17(21.0 \%)$ & $21(26.3 \%)$ & $5(9.6 \%)$ \\
Carotid disease & $16(21.9 \%)$ & $20(24.7 \%)$ & $7(7.5 \%)$ & $7(21.2 \%)$ \\
Peripheral arterial disease & $4(5.5 \%)$ & $34(8.6 \%)$ & $33(41.8 \%)$ & $20(38.5 \%)$ \\
Diabetes mellitus & $25(34.2 \%)$ & $34(42.0 \%)$ & & $20.5 \%)$ \\
\hline
\end{tabular}

TABLe 2: Clinical testing.

\begin{tabular}{|c|c|c|c|c|c|}
\hline & $\begin{array}{l}\text { Distress index }<10 \\
\quad(n=73)\end{array}$ & $\begin{array}{l}\text { Distress index } 10-20 \\
(n=81)\end{array}$ & $\begin{array}{l}\text { Distress index } 20-30 \\
\quad(n=80)\end{array}$ & $\begin{array}{c}\text { Distress index }>30 \\
\quad(n=52)\end{array}$ & $P$ value \\
\hline \multicolumn{6}{|l|}{ Echocardiogram } \\
\hline Aortic valve area $\left(\mathrm{cm}^{2}\right)$ & $0.71 \pm 0.17$ & $0.71 \pm 0.20$ & $0.77 \pm 0.21$ & $0.76 \pm 0.19$ & 0.146 \\
\hline Aortic valve area index $\left(\mathrm{cm}^{2} / \mathrm{m}^{2}\right)$ & $0.38 \pm 0.10$ & $0.38 \pm 0.11$ & $0.45 \pm 0.39$ & $0.43 \pm 0.12$ & 0.134 \\
\hline Left ventricular ejection fraction (\%) & $56 \pm 17$ & $54 \pm 14$ & $54 \pm 15$ & $58 \pm 15$ & 0.623 \\
\hline \multicolumn{6}{|l|}{ Gated computed tomography } \\
\hline Aortic annulus area $\left(\mathrm{mm}^{2}\right)$ & $447 \pm 75$ & $468 \pm 95$ & $440 \pm 101$ & $408 \pm 109$ & 0.010 \\
\hline Aortic annulus perimeter $(\mathrm{mm})$ & $77 \pm 10$ & $79 \pm 9$ & $77 \pm 9$ & $73 \pm 11$ & 0.018 \\
\hline
\end{tabular}

The association between socioeconomic status and health outcomes, particularly with cardiovascular disease, has been well documented [6]. Low SES has been linked with a higher prevalence of multiple cardiovascular conditions, including AS [7], rheumatic heart disease [8], and abdominal aortic aneurysm (AAA) [9], as well as worse morbidity and/ or mortality in the setting of aortic aneurysm [10], aortic dissection [11], stable coronary artery disease [12], and acute coronary syndromes $[13,14]$. Low SES has also been associated with delayed and/or absent referral for cardiac 
TABle 3: Procedural information.

\begin{tabular}{|c|c|c|c|c|}
\hline & $\begin{array}{c}\text { Distress index }<10 \\
\quad(n=73)\end{array}$ & $\begin{array}{l}\text { Distress index } 10-20 \\
(n=81)\end{array}$ & $\begin{array}{l}\text { Distress index } 20-30 \\
\quad(n=80)\end{array}$ & $\begin{array}{c}\text { Distress index }>30 \\
(n=52)\end{array}$ \\
\hline Conscious sedation & $64(87.7 \%)$ & $61(75.3 \%)$ & $61(77.2 \%)$ & $37(71.2 \%)$ \\
\hline Transfemoral access & $73(100.0 \%)$ & $81(100.0 \%)$ & $79(98.8 \%)$ & $52(100.0 \%)$ \\
\hline Predilatation & $61(83.6 \%)$ & $65(80.2 \%)$ & $52(65.0 \%)$ & $35(67.3 \%)$ \\
\hline \multicolumn{5}{|l|}{ Transcatheter value type } \\
\hline Edwards SAPIEN 3 & $58(79.5 \%)$ & $65(80.2 \%)$ & $59(73.8 \%)$ & $42(80.8 \%)$ \\
\hline Medtronic Evolut R/PRO & $15(20.5 \%)$ & $16(19.8 \%)$ & $21(26.3 \%)$ & $10(19.2 \%)$ \\
\hline \multicolumn{5}{|l|}{ Transcatheter valve size } \\
\hline $20 \mathrm{~mm}$ & $5(6.8 \%)$ & $2(2.5 \%)$ & $2(2.5 \%)$ & $6(11.5 \%)$ \\
\hline $23 \mathrm{~mm}$ & $20(27.4 \%)$ & $23(28.4 \%)$ & $25(31.3 \%)$ & $21(4.4 \%)$ \\
\hline $26 \mathrm{~mm}$ & $33(45.2 \%)$ & $30(37.0 \%)$ & $31(38.8 \%)$ & $20(38.5 \%)$ \\
\hline $29 \mathrm{~mm}$ & $14(19.2 \%)$ & $25(30.9 \%)$ & $19(23.8 \%)$ & $2(3.8 \%)$ \\
\hline $34 \mathrm{~mm}$ & $1(1.4 \%)$ & $1(1.2 \%)$ & $3(3.8 \%)$ & $3(5.8 \%)$ \\
\hline Postdilatation & $3(4.1 \%)$ & $4(4.9 \%)$ & $5(6.3 \%)$ & $1(1.9 \%)$ \\
\hline
\end{tabular}

TABLE 4: Discharge information.

\begin{tabular}{|c|c|c|c|c|c|}
\hline & $\begin{array}{c}\text { Distress index }<10 \\
\quad(n=73)\end{array}$ & $\begin{array}{c}\text { Distress index } 10-20 \\
(n=81)\end{array}$ & $\begin{array}{l}\text { Distress index } 20-30 \\
\quad(n=80)\end{array}$ & $\begin{array}{c}\text { Distress index }>30 \\
(n=52)\end{array}$ & $P$ value \\
\hline \multicolumn{6}{|l|}{ Discharge prescriptions } \\
\hline Aspirin & $70(95.9 \%)$ & $71(89.9 \%)$ & $76(96.2 \%)$ & $49(96.1 \%)$ & 0.254 \\
\hline Clopidogrel & $44(60.3 \%)$ & $49(62.0 \%)$ & $49(62.0 \%)$ & $35(68.6 \%)$ & 0.806 \\
\hline Ticagrelor & $0(0 \%)$ & $2(2.5 \%)$ & $1(1.3 \%)$ & $4(7.8 \%)$ & 0.038 \\
\hline Warfarin & $16(21.9 \%)$ & $21(26.6 \%)$ & $14(17.7 \%)$ & $10(19.6 \%)$ & 0.576 \\
\hline Apixaban & $6(8.2 \%)$ & $5(6.3 \%)$ & $9(11.4 \%)$ & $1(2.0 \%)$ & 0.239 \\
\hline Rivaroxaban & $2(2.7 \%)$ & $1(1.3 \%)$ & $2(2.5 \%)$ & $1(2.0 \%)$ & 0.923 \\
\hline Dabigatran & $3(4.1 \%)$ & $1(1.3 \%)$ & $3(3.8 \%)$ & $0(0 \%)$ & 0.370 \\
\hline Discharge location & & & & & 0.545 \\
\hline Home & $58(79.5 \%)$ & 63 (79.7\%) & 69 (87.3\%) & $42(82.4 \%)$ & \\
\hline Skilled nursing facility & $15(20.5 \%)$ & $16(20.3 \%)$ & $10(12.7 \%)$ & $9(17.6 \%)$ & \\
\hline \multicolumn{6}{|l|}{ Length of stay (days) } \\
\hline Admission to discharge & $5.1 \pm 5.3$ & $5.7 \pm 6.6$ & $5.7 \pm 5.1$ & $6.2 \pm 6.7$ & 0.776 \\
\hline TAVR procedure to discharge & $3.1 \pm 2.1$ & $8.0 \pm 41.1$ & $3.2 \pm 2.6$ & $3.3 \pm 4.0$ & 0.424 \\
\hline \multicolumn{6}{|l|}{ In-hospital } \\
\hline Major adverse cardiac events & $4(5.5 \%)$ & $10(12.3 \%)$ & $7(8.8 \%)$ & $3(5.8 \%)$ & 0.402 \\
\hline All-cause mortality & $0(0 \%)$ & $2(2.5 \%)$ & $1(1.3 \%)$ & $1(1.9 \%)$ & 0.609 \\
\hline Disabling stroke & $2(2.7 \%)$ & $1(1.2 \%)$ & $0(0 \%)$ & $0(0 \%)$ & 0.329 \\
\hline New pacemaker & $2(3.3 \%)$ & $8(10.8 \%)$ & $6(9.0 \%)$ & $2(4.2 \%)$ & 0.282 \\
\hline \multicolumn{6}{|c|}{ 30-day (includes in-hospital outcomes) } \\
\hline Major adverse cardiac events & $4(5.5 \%)$ & $14(17.3 \%)$ & $10(12.5 \%)$ & $4(7.7 \%)$ & 0.102 \\
\hline All-cause mortality & $0(0 \%)$ & $2(2.5 \%)$ & $1(1.3 \%)$ & $2(3.8 \%)$ & 0.397 \\
\hline Disabling stroke & $2(2.8 \%)$ & $2(2.5 \%)$ & $1(1.3 \%)$ & $0(0 \%)$ & 0.643 \\
\hline New pacemaker & $2(3.3 \%)$ & $11(15.5 \%)$ & $8(12.1 \%)$ & $2(4.3 \%)$ & 0.055 \\
\hline All-cause readmission & $11(15.3 \%)$ & $21(26.9 \%)$ & $21(26.6 \%)$ & $10(20.0 \%)$ & 0.267 \\
\hline
\end{tabular}

procedures $[15,16]$ as well as poor outcomes following multiple cardiovascular interventions including PCI [17-19], CABG [20-22], aortic and/or mitral valve surgery [21, 23-25], infrainguinal bypass [26, 27], and aortic aneurysm repair [28-30].

Few studies have examined the association of SES with referral for TAVR referral and/or outcomes [31, 32]. One study examining data from the New York State Department Statewide Planning and Research Cooperative System demonstrated that the proportion of TAVR procedures performed in patients from low-income areas increased over time while that in high-income areas decreased over time, suggesting a resolution of health disparities over time due to penetration of the new technology [31]. Nathan A. et al. recently presented data, however, which suggest that TAVR programs may be concentrated in wealthier areas raising questions about access (Nathan A. et al. Stable Ischemic Heart Disease and TAVR. Presented at the Society for Cardiovascular Angiography and Interventions Scientific Session; April 28-May 1, 2021 (virtual meeting)).

DCI has been associated with higher rates of postoperative morbidity and mortality following CABG [33, 34]. One study demonstrated that, for every 25-point increase in the DCI, the risk-adjusted mortality following CABG increased 14\% [34]. While our study did not demonstrate any association between the DCI and outcomes in patients 


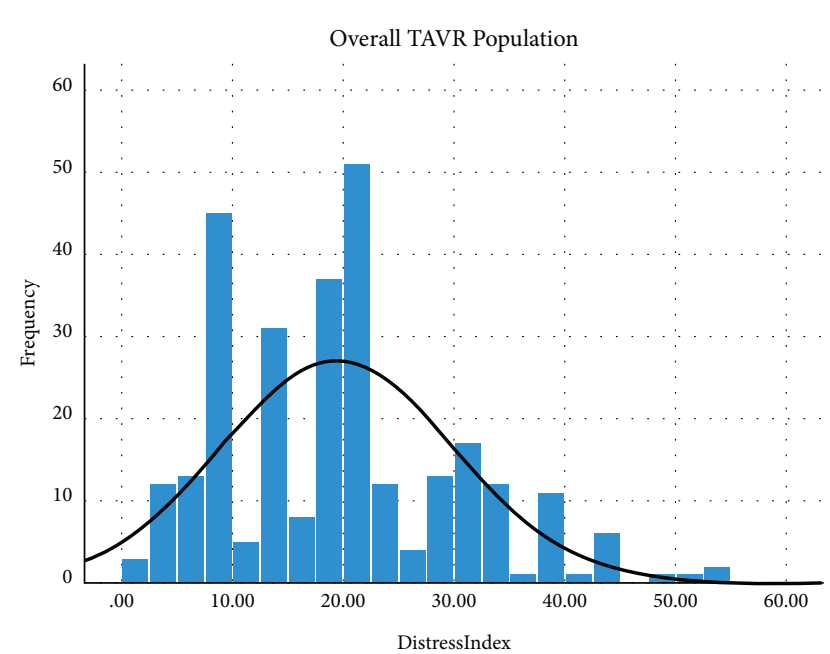

Mean $=19.41$

Std. Dev. $=10.585$

$\mathrm{N}=286$

(a)

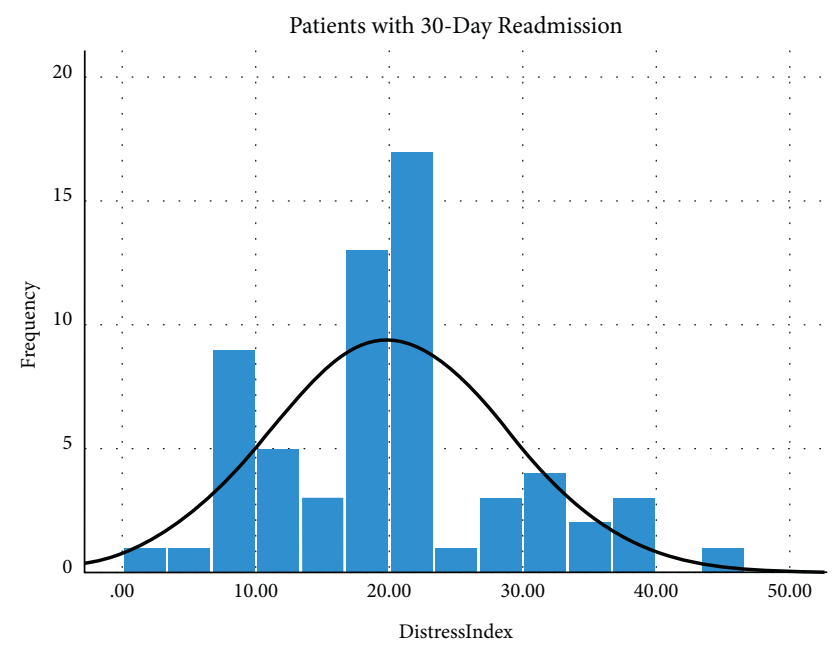

Mean $=19.95$

Std. Dev. $=8.944$

$\mathrm{N}=63$

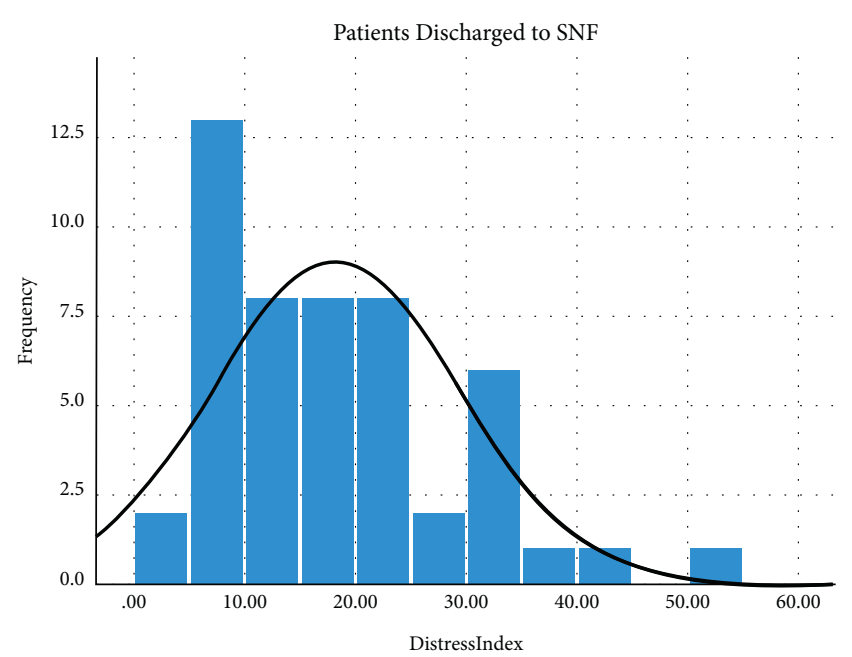

Mean $=18.27$ Std. Dev. $=11.036$ $\mathrm{N}=50$

(b)

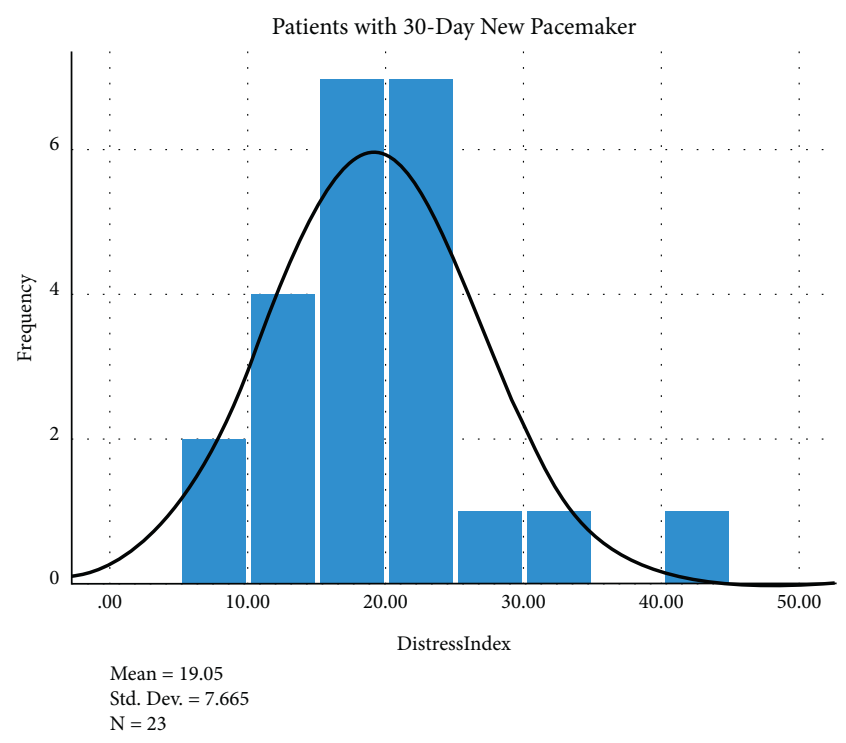

(d)

(c)

FIGURE 2: Histogram of the distressed communities index of our study population for TAVR patients (a) overall, (b) requiring a skilled nursing facility, (c) requiring a new pacemaker, and (d) presenting with 30-day readmission.

undergoing TAVR, there was a higher rate of high-risk features in the highest DCI group, including worse renal function and smaller aortic annuli.

Our study had a number of limitations. First, observational data in this study were not centrally adjudicated but rather internally validated. Second, our study comprised patients undergoing TAVR, and so, patients who were not referred for TAVR were not captured. Third, the distribution of the DCI in Suffolk County is not generalizable to other regions as Suffolk County, along with its neighboring county of Nassau, is among the most affluent areas in the state of New York. As a result, we are unable to compare differences in truly distressed (i.e., DCI $>80$ ) versus prosperous (i.e., DCI $<20)$ areas, and so, we were only able to compare the most affluent versus the least affluent areas of the region. Fourth, ethnic and racial data were not captured in this study [35-38]. The uneven distribution with which TAVR reaches certain racial and ethnic groups has been widely acknowledged $[38,39]$. Finally, there may be unknown confounding variables contributing to the associations reported in this study, and the numbers are small so that a type II error cannot be completely excluded.

When innovative technologies (i.e., TAVR) penetrate a specific population, the relationship between socioeconomic disparities and health outcomes can be variable. This prospective observational study of adults with severe symptomatic AS or bioprosthetic aortic valve degeneration suggests that once detected and referred for TAVR, 


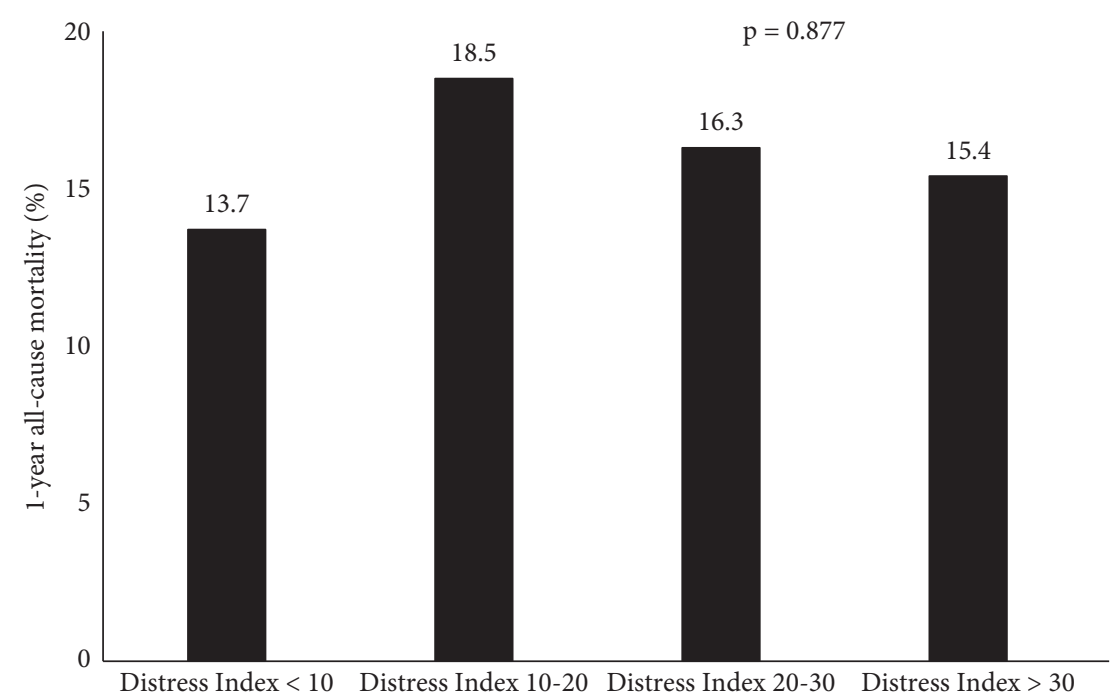

FIGURE 3: Rates of 1-year all-cause mortality categorized by the distressed communities index group.

TABLE 5: TAVR/SAVR in New York state.

\begin{tabular}{cccccccc}
\hline & & & & & & TAVR \\
Year & Total & SAVR total & Elective & Urgent/emergent & TAVR total & Elective & Urgent/emergent \\
\hline 2015 & 5990 & $3856(64.4 \%)$ & $2553(66.2 \%)$ & $1303(33.8 \%)$ & $2134(35.6 \%)$ & $1455(68.2 \%)$ & $679(31.8 \%)$ \\
2016 & 7094 & $3746(67.6 \%)$ & $2531(67.6 \%)$ & $1215(32.4 \%)$ & $3348(47.2 \%)$ & $2451(73.2 \%)$ & $897(26.8 \%)$ \\
2017 & 7074 & $3101(43.8 \%)$ & $2156(69.5 \%)$ & $945(30.6 \%)$ & $3973(56.2 \%)$ & $2988(75.2 \%)$ & $985(24.8 \%)$ \\
2018 & 6911 & $2841(41.1 \%)$ & $1912(67.3 \%)$ & $929(32.7 \%)$ & $4070(58.9 \%)$ & $3166(77.8 \%)$ & $904(22.2 \%)$ \\
\hline
\end{tabular}

differences in outcomes across the DCI remain similar rendering access and case selection, the likely discriminators.

\section{Data Availability}

The distressed communities index can be found at eig.org, the Suffolk County demographic data can be found at newyork-demographics.com, and state wide TAVI data can be obtained from the SPARCS database.

\section{Conflicts of Interest}

The authors declare that they have no conflicts of interest.

\section{References}

[1] C. Sorenson, R. Tarricone, M. Siebert, and M. Drummond, "Applying health economics for policy decision making: do devices differ from drugs? Europace: European pacing, arrhythmias, and cardiac electrophysiology," Journal of the Working Groups on Cardiac Pacing, Arrhythmias, and Cardiac Cellular Electrophysiology of the European Society of Cardiology, vol. 13, no. Suppl 2, pp. ii54-ii58, 2011.

[2] Distressed Communities Index.

[3] H. Kundi, J. B. Strom, L. R. Valsdottir et al., "Trends in isolated surgical aortic valve replacement according to hospital-based transcatheter aortic valve replacement volumes," JACC: Cardiovascular Interventions, vol. 11, no. 21, pp. 2148-2156, 2018.

[4] Suffolk County Demographics.
[5] N. D. Desai, S. M. O’Brien, D. J. Cohen et al., "Composite metric for benchmarking site performance in transcatheter aortic valve replacement," Circulation, vol. 144, no. 3, pp. 186-194, 2021.

[6] D. A. Alter, C. D. Naylor, P. Austin, and J. V. Tu, "Effects of socioeconomic status on access to invasive cardiac procedures and on mortality after acute myocardial infarction," New England Journal of Medicine, vol. 341, no. 18, pp. 1359-1367, 1999.

[7] P. Andell, X. Li, A. Martinsson et al., "Neighborhood socioeconomic status and aortic stenosis: a Swedish study based on nationwide registries and an echocardiographic screening cohort," International Journal of Cardiology, vol. 318, pp. 153-159, 2020.

[8] L. Baro, N. Sharma, D. Toor et al., "A hospital-based study of socioeconomic status and clinical spectrum of rheumatic heart disease patients of Assam, North-East India," European Journal of Preventive Cardiology, vol. 25, no. 12, pp. 13031306, 2018.

[9] M. Zarrouk, J. Holst, M. Malina et al., "The importance of socioeconomic factors for compliance and outcome at screening for abdominal aortic aneurysm in 65-year-old men," Journal of Vascular Surgery, vol. 58, no. 1, pp. 50-55, 2013.

[10] T. Ohrlander, J. Merlo, H. Ohlsson, B. Sonesson, and S. Acosta, "Socioeconomic position, comorbidity, and mortality in aortic aneurysms: a 13-year prospective cohort study," Annals of Vascular Surgery, vol. 26, no. 3, pp. 312-321, 2012.

[11] L. S. Kabbani, S. Wasilenko, T. J. Nypaver et al., "Socioeconomic disparities affect survival after aortic dissection," Journal of Vascular Surgery, vol. 64, no. 5, pp. 1239-1245, 2016. 
[12] R. K. Wadhera, D. L. Bhatt, A. J. H. Kind et al., "Association of outpatient practice-level socioeconomic disadvantage with quality of care and outcomes among older adults with coronary artery disease: implications for value-based payment," Circulation. Cardiovascular Quality and Outcomes, vol. 13, Article ID e005977, 2020.

[13] C. Blais, D. Hamel, and S. Rinfret, "Impact of socioeconomic deprivation and area of residence on access to coronary revascularization and mortality after a first acute myocardial infarction in québec," Canadian Journal of Cardiology, vol. 28, no. 2, pp. 169-177, 2012.

[14] K. Hyun, J. Redfern, M. Woodward et al., "Socioeconomic equity in the receipt of in-hospital care and outcomes in Australian acute coronary syndrome patients: the CONCORDANCE registry," Heart Lung \& Circulation, vol. 27, no. 12, pp. 1398-1405, 2018.

[15] G. E. Fabreau, A. A. Leung, D. A. Southern et al., "Sex, socioeconomic status, access to cardiac catheterization, and outcomes for acute coronary syndromes in the context of universal healthcare coverage," Circulation: Cardiovascular Quality and Outcomes, vol. 7, no. 4, pp. 540-549, 2014.

[16] C. M. Yong, F. Abnousi, S. M. Asch, and P. A. Heidenreich, "Socioeconomic inequalities in quality of care and outcomes among patients with acute coronary syndrome in the modern era of drug eluting stents," Journal of the American Heart Association, vol. 3, Article ID e001029, 2014.

[17] K. Manderbacka, M. Arffman, S. Lumme, and I. Keskimäki, "Are there socioeconomic differences in outcomes of coronary revascularizations-a register-based cohort study," The European Journal of Public Health, vol. 25, no. 6, pp. 984-989, 2015.

[18] D. A. Jones, J. P. Howard, K. S. Rathod et al., "The impact of socio-economic status on all-cause mortality after percutaneous coronary intervention: an observational cohort study of 13,770 patients," EuroIntervention, vol. 10, no. 11, pp. e1-e8, 2015.

[19] A. Shimony, D. Zahger, R. Ilia, A. Shalev, and C. Cafri, "Impact of the community's socioeconomic status on characteristics and outcomes of patients undergoing percutaneous coronary intervention," International Journal of Cardiology, vol. 144 , no. 3 , pp. $379-382,2010$.

[20] G. N. Coyan, A. Okoye, A. Shah et al., "Effect of neighborhood socioeconomic factors on readmissions and mortality after coronary artery bypass grafting," The Annals of Thoracic Surgery, vol. 111, no. 2, pp. 561-567, 2021.

[21] C. G. Koch, L. Li, G. A. Kaplan et al., "Socioeconomic position, not race, is linked to death after cardiac surgery," Circulation: Cardiovascular Quality and Outcomes, vol. 3, no. 3, pp. 267-276, 2010.

[22] D. A. M. Dzayee, T. Ivert, O. Beiki, L. Alfredsson, R. Ljung, and T. Moradi, "Short and long term mortality after coronary artery bypass grafting (CABG) is influenced by socioeconomic position but not by migration status in Sweden, 1995-2007," PLoS One, vol. 8, no. 5, Article ID e63877, 2013.

[23] J. P. Bagger, M.-B. Edwards, and K. M. Taylor, "Influence of socioeconomic status on survival after primary aortic or mitral valve replacement," Heart, vol. 94, no. 2, pp. 182-185, 2008.

[24] T. R. Feng, M. M. Hoyler, X. Ma, L. Q. Rong, and R. S. White, "Insurance status and socioeconomic markers affect readmission rates after cardiac valve surgery," Journal of
Cardiothoracic and Vascular Anesthesia, vol. 34, no. 3, pp. 668-678, 2020.

[25] M. M. Hoyler, T. R. Feng, X. Ma et al., "Insurance status and socioeconomic factors affect early mortality after cardiac valve surgery," Journal of Cardiothoracic and Vascular Anesthesia, vol. 34, no. 12, pp. 3234-3242, 2020.

[26] R. B. Hawkins, E. J. Charles, J. H. Mehaffey et al., "Socioeconomic Distressed Communities Index associated with worse limb-related outcomes after infrainguinal bypass," Journal of Vascular Surgery, vol. 70, no. 3, pp. 786-794, 2019.

[27] R. B. Hawkins, J. H. Mehaffey, E. J. Charles, J. A. Kern, E. B. Schneider, and M. C. Tracci, "Socioeconomically Distressed Communities Index independently predicts major adverse limb events after infrainguinal bypass in a national cohort," Journal of Vascular Surgery, vol. 70, no. 6, pp. 1985-1993, 2019.

[28] M. Khashram, S. Pitama, J. A. Williman, G. T. Jones, and J. A. Roake, "Survival disparity following abdominal aortic aneurysm repair highlights inequality in ethnic and socioeconomic status," European Journal of Vascular and Endovascular Surgery, vol. 54, no. 6, pp. 689-696, 2017.

[29] M. D. Perlstein, S. Gupta, X. Ma, L. Q. Rong, G. Askin, and R. S. White, "Abdominal aortic aneurysm repair readmissions and disparities of socioeconomic status: a multistate analysis, 2007-2014," Journal of Cardiothoracic and Vascular Anesthesia, vol. 33, no. 10, pp. 2737-2745, 2019.

[30] Z. Al Adas, T. J. Nypaver, A. D. Shepard et al., "Survival after abdominal aortic aneurysm repair is affected by socioeconomic status," Journal of Vascular Surgery, vol. 69, no. 5, pp. 1437-1443, 2019.

[31] L. Q. Rong, J. Mao, A. R. Sedrakyan et al., "Changes in the socioeconomic status of patients receiving TAVR in New York State," Journal of Cardiac Surgery, vol. 35, no. 1, pp. 54-57, 2020.

[32] A. Sleder, S. Tackett, M. Cerasale et al., "Socioeconomic and racial disparities: a case-control study of patients receiving transcatheter aortic valve replacement for severe aortic stenosis," Journal of Racial and Ethnic Health Disparities, vol. 4, no. 6, pp. 1189-1194, 2017.

[33] E. J. Charles, J. H. Mehaffey, R. B. Hawkins et al., "Socioeconomic distressed communities index predicts risk-adjusted mortality after cardiac surgery," The Annals of Thoracic Surgery, vol. 107, no. 6, pp. 1706-1712, 2019.

[34] J. H. Mehaffey, R. B. Hawkins, E. J. Charles et al., "Distressed communities are associated with worse outcomes after coronary artery bypass surgery," The Journal of Thoracic and Cardiovascular Surgery, vol. 160, no. 2, pp. 425-432, 2020.

[35] V. T. Nkomo, J. M. Gardin, T. N. Skelton, J. S. Gottdiener, C. G. Scott, and M. Enriquez-Sarano, "Burden of valvular heart diseases: a population-based study," The Lancet, vol. 368, no. 9540, pp. 1005-1011, 2006.

[36] D. K. Patel, K. D. Green, M. Fudim, F. E. Harrell, T. J. Wang, and M. A. Robbins, "Racial differences in the prevalence of severe aortic stenosis," Journal of the American Heart Association, vol. 3, Article ID e000879, 2014.

[37] W. S. Aronow, C. Ahn, and I. Kronzon, "Comparison of echocardiographic abnormalities in African-American, Hispanic, and white men and women aged $>60$ years," The American Journal of Cardiology, vol. 87, no. 9, pp. 1131-1133, 2001.

[38] J. Matthew Brennan, M. B. Leon, P. Sheridan et al., "Racial differences in the use of aortic valve replacement for treatment of symptomatic severe aortic valve stenosis in the 
transcatheter aortic valve replacement era," Journal of the American Heart Association, vol. 9, Article ID e015879, 2020.

[39] W. Batchelor, S. Anwaruddin, L. Ross et al., "Aortic valve stenosis treatment disparities in the underserved," Journal of the American College of Cardiology, vol. 74, no. 18, pp. 2313-2321, 2019. 